\title{
OPEN Defect induced ferromagnetic ordering and room temperature negative magnetoresistance in MoTeP
}

\author{
Debarati Pal ${ }^{1}$, Shiv Kumar ${ }^{2}$, Prashant Shahi ${ }^{3}$, Sambhab Dan ${ }^{1}$, Abhineet Verma ${ }^{6}$, \\ Vinod K. Gangwar ${ }^{1}$, Mahima Singh ${ }^{1}$, Sujoy Chakravarty ${ }^{4}$, Yoshiya Uwatoko ${ }^{5}$, Satyen Saha ${ }^{6}$, \\ Swapnil Patil ${ }^{1 \rrbracket}$ \& Sandip Chatterjee ${ }^{1 \bowtie}$
}

The magneto-transport, magnetization and theoretical electronic-structure have been investigated on type-II Weyl semimetallic MoTeP. The ferromagnetic ordering is observed in the studied sample and it has been shown that the observed magnetic ordering is due to the defect states. It has also been demonstrated that the presence of ferromagnetic ordering in effect suppresses the magnetoresistance (MR) significantly. Interestingly, a change-over from positive to negative MR is observed at higher temperature which has been attributed to the dominance of spin scattering suppression.

The realization of the Weyl semimetals (WSMs) has sparked extreme research interests in condensed-matter physics community since it provides the recognition of the Weyl fermions. This topological semimetal is associated with the lack of time-reversal or inversion symmetry. The concept of WSMs can be categorized into two ways. In type-I WSMs, the linear nondegenerate band crossings lead to a point like Fermi surface (FS) when the chemical potential is adjusted to the energy of Weyl Points (WPs). The hole and electron pockets form the $\mathrm{WPs}^{1,2}$ in the type-II WSMs unlike the case of type-I in which band crossings produce the WPs. A finite density of states at the chemical potential is created due to overlapping of these electron and hole pockets over a range of energies $^{3-5}$. Again the type-I WSM obeys Lorentz invariance, in contrast, type-II does not. These Weyl points are twofold degenerate and always come in pairs with opposite chirality, namely, a source and a sink of the Berry curvature $^{6,7}$. The concept of type-II WSMs was brought forward by studying the topological properties of $\mathrm{MoTe}_{2}$, $\mathrm{WTe}_{2}$ and their alloy $\mathrm{Mo}_{1-\mathrm{x}} \mathrm{W}_{\mathrm{x}} \mathrm{Te}_{2}{ }^{8-10}$. With time many researches were devoted to $2 \mathrm{D}$ transition metal dichalcogenides materials (TMDs) with chemical formula $\mathrm{MX}_{2}$, where $\mathrm{M}$ is a transition metal and $\mathrm{X}$ is a chalcogen atom (S, Se or Te) due to their significant electronic and optoelectronic properties. Very recently, 3D TMDs $\mathrm{WP}_{2}$ and $\mathrm{MoP}_{2}$ were predicted to host four pairs of type-II Weyl points below the Fermi energy with a unique feature of having same chirality for the nearest WPs ${ }^{11}$.

In addition, WSMs are in general renowned for their negative longitudinal magnetoresistance (NLMR) induced by chiral anomaly ${ }^{12,13}$, which refers to the non-conservation of chiral charge around the Weyl nodes when applied electric and magnetic fields are non-orthogonal $(\mathrm{E} . \mathrm{B} \neq 0)$. The experimental measurement of NLMR is very sensitive, and especially for type-II WSMs, the NLMR can only be observed along specific crystalline directions and in samples with appropriate chemical potential ${ }^{14,15}$. In type-I Weyl semimetals, the chiral anomaly always appears regardless of the direction of the applied magnetic fields. Thus the observation of positive longitudinal magnetoresistance under applied magnetic field perpendicular to electric field is strong evidence to distinguish type-II WSMs from type-I WSMs. To best of our knowledge, so far, the NLMR has not been reported for $\mathrm{MoTe}_{2}{ }^{16}$.

Furthermore, there are many reports on the spin-orbit coupling and the interesting consequences of electrical and optical properties in these systems. However, there are very limited, and mostly theoretical studies on intrinsic magnetism based on monolayer structure calculation ${ }^{17-20}$. Theoretical and experimental work shows

\footnotetext{
${ }^{1}$ Department of Physics, Indian Institute of Technology (Banaras Hindu University), Varanasi 221005, India. ${ }^{2}$ Hiroshima Synchrotron Radiation Center, Hiroshima University, Higashi-Hiroshima City 739-0046, Japan. ${ }^{3}$ Department of Physics, D.D.U. Gorakhpur University, Gorakhpur 273009, India. ${ }^{4}$ UGC-DAE Consortium

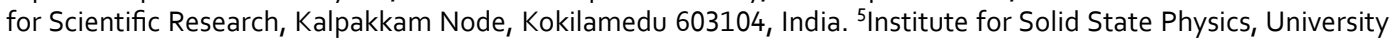
of Tokyo, Kashiwa, Chiba 277-8581, Japan. ${ }^{6}$ Department of Chemistry, Institute of Science (Banaras Hindu University), Varanasi 221005, India.『email: spatil.phy@itbhu.ac.in; schatterji.app@iitbhu.ac.in
} 
that in the absence of crystalline imperfections, the Mo-based TMDs are nonmagnetic ${ }^{21-23}$. Therefore, by adding defects one may induce magnetism into these materials and this ability can open up a host of new opportunities for spintronic applications. In this report we have investigated for the first time the type-II Weyl semimetallic MoTeP. Our study indicates that it is a defect induced magnetic Weyl semimetallic material.

\section{Methods}

Sample preparation. Single crystals of MoTeP were grown by the chemical vapor transport method ${ }^{24,25}$ in 3 steps. In the first step polycrystal of MoTeP was synthesized by heating stoichiometric amounts of Mo, Te and P powders at $800^{\circ} \mathrm{C}$ for $24 \mathrm{~h}$. This MoTeP polycrystal was again sealed in an evacuated quartz ampoule with iodine as a transport agent. The ampoule is put in the two-zone furnace with a temperature gradient $1050{ }^{\circ} \mathrm{C}$ (source) to $950^{\circ} \mathrm{C}$ (sink) for two weeks and then quenched in ice-cold water. This fast cooling process yields $1 \mathrm{~T}^{\prime}$-phase of MoTeP single crystal.

Structural characterization. Figure S1 of supplementary shows the room temperature X-ray diffraction (XRD) pattern of the MoTeP material. We have performed Le-Bail refinement of the powder XRD data $\left(\mathrm{CuK}_{\alpha}\right.$ radiation) of MoTeP single crystal to obtain the lattice parameters. The extracted lattice parameters are $a=3.3759(52) \AA, b=6.2907(93) \AA$ and $c=13.8629(28) \AA$ whereas the calculated $\alpha, \beta$ and $\gamma$ values are $90^{\circ}, 94.82^{\circ}$ and $90^{\circ}$. The obtained lattice parameters are consistent with $1 \mathrm{~T}^{\prime}\left(\mathrm{P} 2_{1} / m\right)$ family of compounds ${ }^{26}$.

Material characterization. Magneto-transport properties measurements were performed in a Quantum Design Physical Properties Measurement System (PPMS) using a conventional four-probe configuration. The measurements were performed in a standard Hall bar geometry i.e. electrical current was applied along the $\mathrm{b}$ axis, and magnetic field perpendicular to the $\mathrm{b}$ axis. Magnetic measurements (both temperature and magnetic field dependent) were performed using quantum design SQUID magnetic properties measurement system (MPMS). Temperature dependent Raman study was executed by Horiba LabRam HR evolution spectrometer. The sample was irradiated with $633 \mathrm{~nm} \mathrm{He-Ne}$ laser. The sample was cooled from 300 to $190 \mathrm{~K}$ by liquid nitrogen when taking Raman measurements at different temperatures.

The density functional theory (DFT) calculations. We performed DFT calculations using ABINIT package ${ }^{27}$ with a projector-augmented-wave (PAW) method. We adopted generalized gradient approximation (GGA) as exchange-correlation proposed by Perdew-Burke-Ernzerhof (PBE). All atoms of MoTeP were fully relaxed with conjugate-gradient algorithm until a force is less than $0.01 \mathrm{eV} \AA^{-1}$ and the energy convergence criteria was put to $10^{-6} \mathrm{eV}$. The electronic calculations were performed using $\Gamma$-centred $\mathrm{K}$-mesh of $15 \times 9 \times 5$ with a plane-wave energy cutoff of $19.1096 \mathrm{Ha}(520 \mathrm{eV})$, spin orbit coupling (SOC) were included in the calculations. We used optimized lattice parameters $(a=3.3418(56) \AA, b=6.0887(71) \AA$ and $c=13.3433(95) \AA$ for the theoretical calculation of MoTeP. For the spin-polarised DOS calculations vacancy and antisite defects were created inside the crystal structure. Additionally, we checked GGA + U with $U=0.0367493 \mathrm{Ha}(1 \mathrm{eV})$, however inclusion of Coulomb interaction $U$ does not affect our results. The output files (.agr) were analysed in XMGRACE ${ }^{28}$ soft- $^{-}$ ware.

\section{Results and discussion}

Zero field resistivity behavior. The temperature evolution of the resistivity $\rho_{\mathrm{xx}}$ shows metallic character throughout the measured temperature range as shown in Fig. 1a. The longitudinal resistivity $\rho_{x x}$ indicating dominant electron-electron and electron-phonon scattering at low temperature as depicted in Fig. 1a inset. MoTeP shows resistivity $36.55 \mu \Omega-\mathrm{cm}$ at $2 \mathrm{~K}$ and this reaches to $963.47 \mu \Omega-\mathrm{cm}$ at $300 \mathrm{~K}$. Thus the residual resistivity ratios $(\mathrm{RRRs})=\rho(300 \mathrm{~K}) / \rho(2 \mathrm{~K})=26.41$ suggesting good crystalline quality of the sample. This value of RRR is consistent with several topological semimetals ${ }^{29-31}$.

The temperature dependence of the resistivity at low temperature can be accounted for by the usual combination of Fermi liquid and electron-phonon scattering mechanisms by fitting to $\rho_{x x}(T)=\rho_{0}+a T^{2}+b T^{5}$, where $\rho_{0}=\rho(T=0 \mathrm{~K})$ with a and $\mathrm{b}$ being fitted parameters (Fig. 1a inset). Three temperature regions $\mathrm{T}_{\mathrm{m} 1}, \mathrm{~T}_{\mathrm{m} 2}, \mathrm{~T}_{\mathrm{m} 3}$ are marked with arrows in Fig. 1b. The observed hysteresis around $\mathrm{T}_{\mathrm{m} 1} \sim 250 \mathrm{~K}$ between the warm-up and the cool-down curves (Fig. 1a) is ascribed to the structural phase transition from the $1 \mathrm{~T}^{\prime}$ to the $\mathrm{T}_{\mathrm{d}}$ structure which is consistent with $\mathrm{MoTe}_{2}{ }^{32,33}$. This structural phase transition is confirmed by temperature dependent Raman spectroscopy (supplementary). The coexistence of $1 \mathrm{~T}^{\prime}$ and $\mathrm{T}_{\mathrm{d}}$ phase is the possible reason behind the hysteresis over a long range of temperature. This type of hysteresis behavior is common in $\mathrm{MoTe}_{2}{ }^{32}$. The $\mathrm{d} \rho_{\mathrm{xx}}(\mathrm{T}) / \mathrm{dT}$ curve exhibited a broad peak around the temperature $\sim 75 \mathrm{~K}$ (marked as $\mathrm{T}_{\mathrm{m} 2}$ ), which suggests the possible change in the electronic structure of $\mathrm{T}_{\mathrm{d}}-\mathrm{MoTeP}$. This is also consistent with the reports on $\mathrm{MoTe}_{2}{ }^{24,34-37}$. However, the temperature $\left(\mathrm{T}_{\mathrm{m} 2}\right)$ for $\mathrm{MoTe}_{2}$ is $\sim 50 \mathrm{~K}$. Temperature dependent mass anisotropy was also reported around $\mathrm{T}_{\mathrm{m} 2}$ in $\mathrm{MoTe}_{2}$ by Chen et al. ${ }^{34}$. We identified the temperature as $\mathrm{T}_{\mathrm{m} 3}$ where 1 st derivative of $\rho_{\mathrm{xx}}$ becomes minimum which is also known as the turn on temperature ${ }^{34}$. Therefore, the observed transport behavior in MoTeP is consistent with

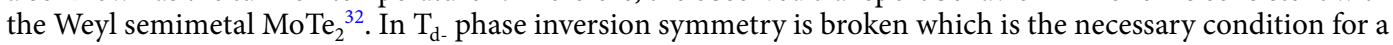
material to have Weyl semimetal phase ${ }^{8,38,39}$ In the present investigation, the existence of $\mathrm{T}_{\mathrm{d}}$-phase in MoTeP is a possibility of this material to be Weyl semimetallic. Furthermore, turn on behavior is commonly attributed to field induced metal insulator transition and is well familiar among extremely large MR (XMR $)^{32,37,40-42}$. Importantly, Q. L. Pei and his group ${ }^{37}$ suggested the electronic structure change (near $50 \mathrm{~K}$ ) as the necessary condition for the presence of the turn-on phenomenon in $\mathrm{WTe}_{2}$ and $\mathrm{T}_{\mathrm{d}}-\mathrm{MoTe}_{2}$. 

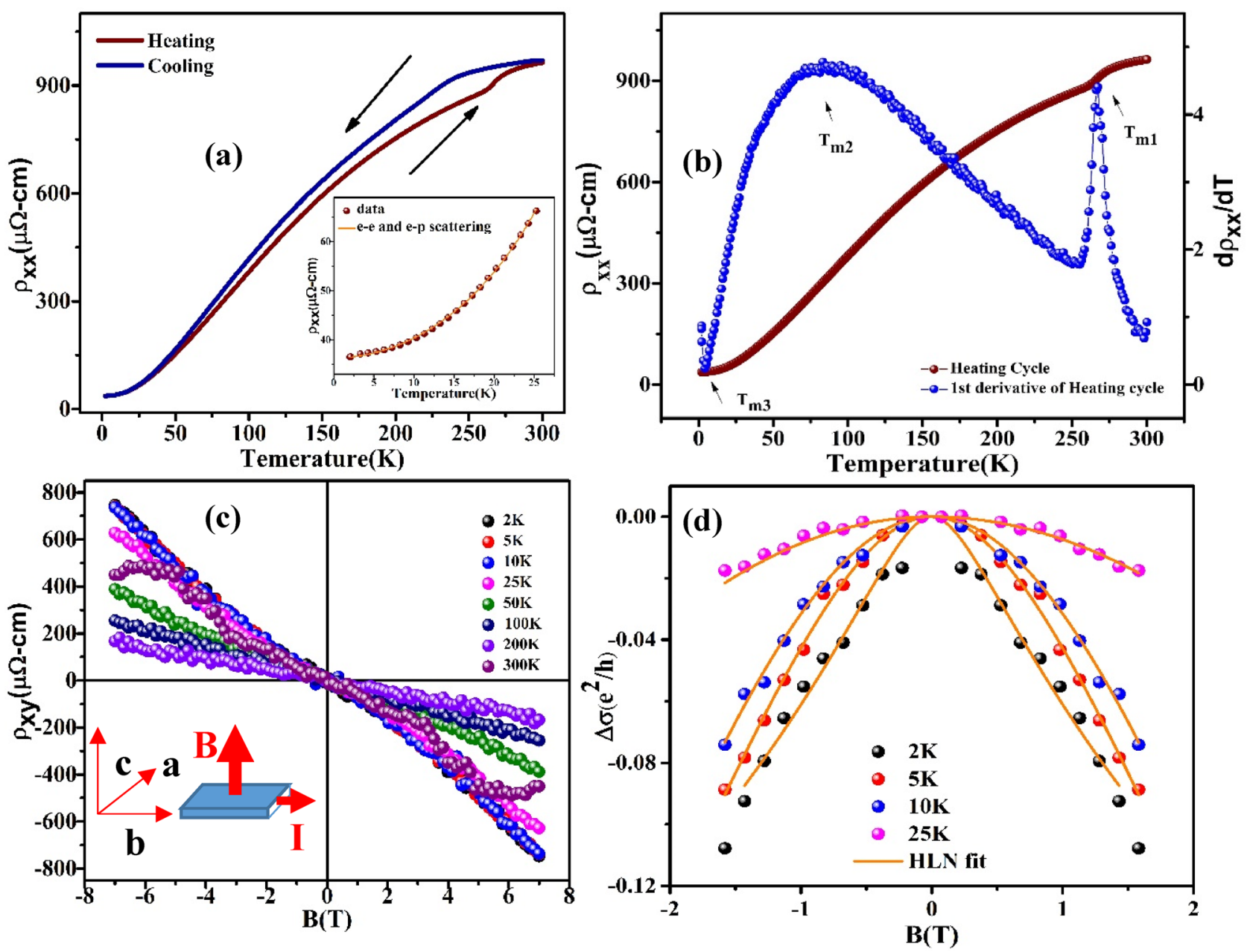

Figure 1. (a) Thermal hysteresis of temperature dependent resistivity of $1 \mathrm{~T}^{\prime} \mathrm{MoTeP}$, inset: low temperature resistivity data, orange solid line is a fit of electron-electron and electron-phonon scattering terms $\rho(T)=\rho_{0}+a T^{2}+b T^{5},(\mathbf{b})$ temperature evolution of $\rho x x$ and $d \rho_{x x} / d T$ at zero magnetic field, (c) Field dependence of Hall data $\rho_{\mathrm{xy}}$ at various temperatures, inset: measurement geometry, (d) low-field low-temperature conductivity data, solid orange line shows HLN fitting to $\Delta \sigma\left(\mathrm{e}^{2} / \mathrm{h}\right)$ at various temperatures.

Hall effect study. Figure 1c displays the magnetic field dependence of Hall resistivity $\rho_{\mathrm{xy}}$. From the Hall resistivity behaviour it is clear that the conduction carriers are dominated by electrons which is consistent to that of other Weyl semimetals ${ }^{43,44}$. However, the $\rho_{x y}(B)$ shows non-linear behavior and with increase of temperature the deviation from linearity of $\rho_{x y}(B)$ increases and finally at $300 \mathrm{~K}$ it shows unusual non-linearity (S-shaped) at high field. This nonlinear $\rho_{\mathrm{xy}}(\mathrm{B})$ implies the existence of both electrons and holes. The S-shaped nonlinearity is similar to that of topological single crystal system $\mathrm{Bi}_{1-\mathrm{x}} \mathrm{Sb}_{\mathrm{x}}{ }^{45}$. In order to determine carrier mobility and carrier density for both type of charge carriers we executed two-carrier model fit with our $\sigma_{x y}$ and $\sigma_{x x}$ data, where the field dependence of the conductivity tensor is given by

$$
\sigma_{x y}=\left[n_{e} \mu_{e}^{2} \frac{1}{1+\left(\mu_{e} B\right)^{2}}-n_{h} \mu_{h}^{2} \frac{1}{1+\left(\mu_{h} B\right)^{2}}\right] \text { eB }
$$

where Hall conductivity

$$
\sigma_{x y}=-\frac{\rho_{x y}}{\rho_{x x}^{2}+\rho_{x y}^{2}}
$$

Here, $n_{e}\left(n_{h}\right)$ and $\mu_{e}\left(\mu_{h}\right)$ are electrons (holes) carrier densities and mobilities, respectively and $\sigma_{x y}$ is the Hall conductivity. Figure $2 \mathrm{a}$ illustrates the temperature dependence of the Hall conductivity and their respective two-band model fit. The fitting of Eq. (1) yields electron and hole densities $0.188 \times 10^{19}$ and $0.182 \times 10^{19} \mathrm{~cm}^{-3}$ respectively at $2 \mathrm{~K}$. The electron and hole mobilities are $2.216 \times 10^{4}$ and $2.196 \times 10^{4} \mathrm{~cm}^{2} / \mathrm{V} \mathrm{s}$. The extracted parameters $n_{e}, n_{h}, \mu_{e}, \mu_{h}$ and their temperature evolution along with a comparative result of $n_{h} / n_{e}$ and $\mu_{h} / \mu_{e}$ is also shown in Fig. $2 \mathrm{c}$,d. This values show that the magnetotransport properties in MoTeP is primarily influenced by electron type charge carriers and a near perfect electron-hole compensation scenario is present in this system at 

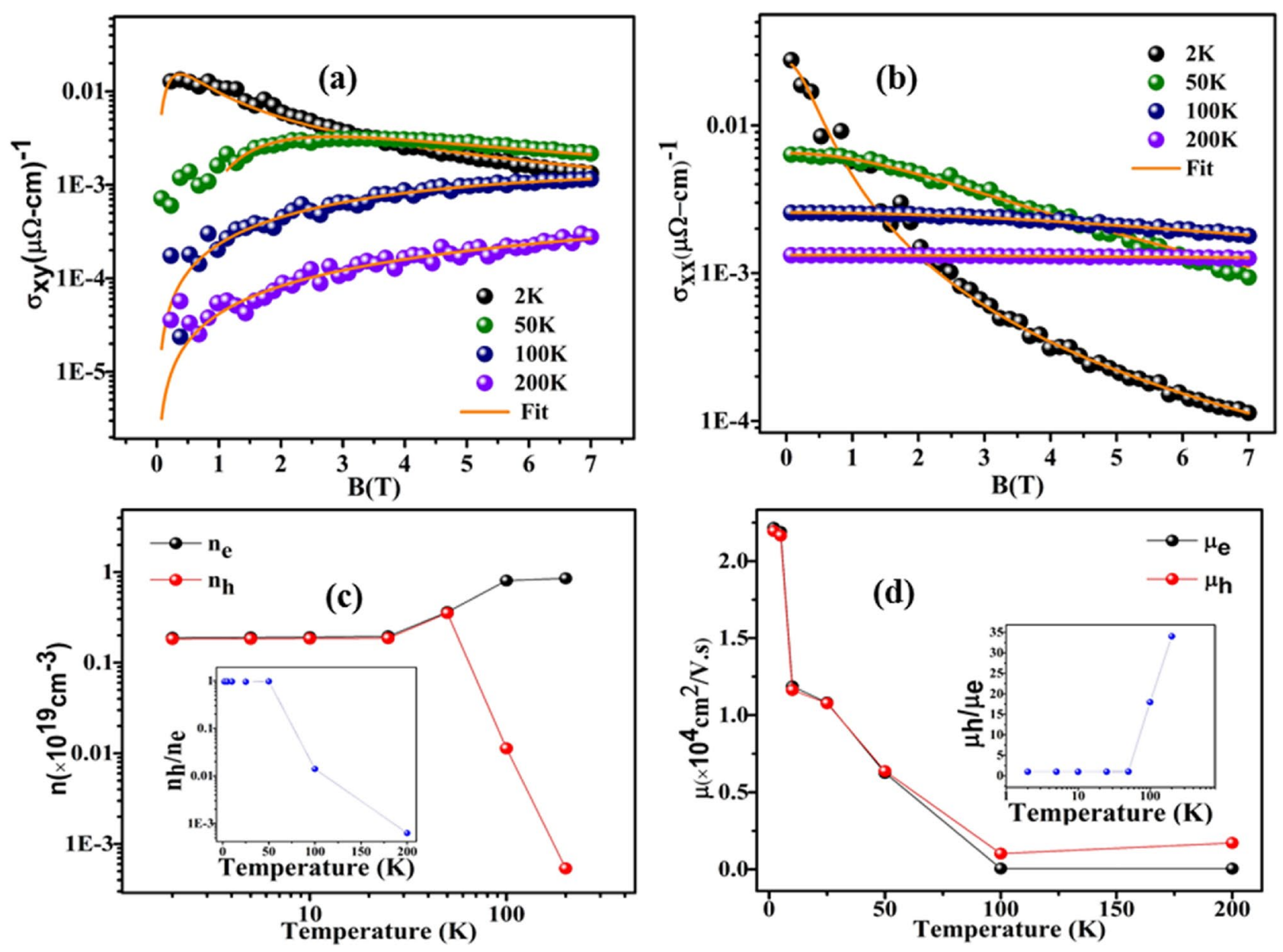

Figure 2. (a,b) Field dependence of Hall conduvtivity $\sigma_{\mathrm{xy}}$ and longitudinal conductivity $\sigma_{\mathrm{xx}}$ at $2,50,100$ and $200 \mathrm{~K}$, orange solid lines are their respective two-carrier model fit with Eqs. (1) and (3), (c) density of electrons $\mathrm{n}_{\mathrm{e}}$ (black circle) and density of hole $\mathrm{n}_{\mathrm{h}}$ ( red circles) as a function of temperature extracted from $\sigma_{\mathrm{xy}}$ inset: ratio of $\mathrm{n}_{\mathrm{h}}$ and $\mathrm{n}_{\mathrm{e}}$ as a function of temperature, (d) electron mobility $\mu_{\mathrm{e}}$ (black circle) and hole mobility $\mu_{\mathrm{h}}$ ( red circles) and their ratio $\mu_{\mathrm{h}} / \mu_{\mathrm{e}}$, inset: as a function of temperature.

low temperature. These values are comparable to many Dirac $\mathrm{Cd}_{3} \mathrm{As}_{2}, \mathrm{ZrTe}_{5}{ }^{46,47}$ and other semimetallic $\mathrm{MoTe}_{2}$, $\mathrm{WTe}_{2}, \mathrm{LaSbTe}, \mathrm{VAl}_{3}{ }^{24,48-50}$ systems. However, our hole mobility is two order less than $\mathrm{WP}_{2}{ }^{42}$. The carrier density is almost constant from 2 to $15 \mathrm{~K}$. However, the mobility of both type of carriers decreases with increase in temperature. Above $50 \mathrm{~K}$ hole mobility and electron density increases rapidly and there is a change in the temperature dependence of the electron and hole density and/or mobility. The electron and hole density extracted at room temperature $\mathrm{n}_{\mathrm{e}} \sim 0.177 \times 10^{19} \mathrm{~cm}^{-3}, \mathrm{n}_{\mathrm{h}} \sim 0.173 \times 10^{19} \mathrm{~cm}^{-3}$ and their mobilities are $\mu_{\mathrm{e}} \sim 0.196 \times 10^{4} \mathrm{~cm}^{2} / \mathrm{V} \mathrm{s}$ and $\mu_{\mathrm{h}} \sim 0.198 \times 10^{4} \mathrm{~cm}^{2} / \mathrm{V}$ s respectively. Furthermore, it is observed that the amplitude of the nonlinear S-shaped becomes flat with increasing $\mathrm{n}_{\mathrm{e}}$. Also, hole plays dominant contribution at this temperature with an increase in $\mu_{\mathrm{h}}$. In order to assess the accuracy of the parameters obtained for charge density and mobility we further fitted our $\sigma_{\mathrm{xx}}$ (Eq. 3) data using

$$
\sigma_{x x}=\left[\frac{n_{e} e \mu_{e}}{1+\left(\mu_{e} B\right)^{2}}+\frac{n_{h} e \mu_{h}}{1+\left(\mu_{h} B\right)^{2}}\right]
$$

where longitudinal conductivity

$$
\sigma_{x x}=\frac{\rho_{x x}}{\rho_{x x^{2}}+\rho_{x y}{ }^{2}}
$$

$\sigma_{\mathrm{xx}}$ is longitudinal resistivity (Fig. 2b) in transverse magnetic field and current configuration. The obtained electron density $\mathrm{n}_{\mathrm{e}} \sim 0.188 \times 10^{19}$ and hole density $\mathrm{n}_{\mathrm{h}} \sim 0.182 \times 10^{19} \mathrm{~cm}^{-3}$ and the extracted electron mobility $\mu_{\mathrm{e}} \sim 2.218 \times 10^{4} \mathrm{~cm}^{2} / \mathrm{V} \mathrm{s}$ and hole mobility $\mu_{\mathrm{h}} \sim 2.164 \times 10^{4} \mathrm{~cm}^{2} / \mathrm{V} \mathrm{s}$ at $2 \mathrm{~K}$. The hole density increases beyond $50 \mathrm{~K}$ whereas and electron-hole density becomes comparable at low temperature. As evident from the Fig. $2 \mathrm{c}$ $\mathrm{n}_{\mathrm{e}}$ increases with temperature whereas $\mathrm{n}_{\mathrm{h}}$ decreases above $50 \mathrm{~K}$. This is similar to $\mathrm{MoTe}_{2}{ }^{34}$. In addition, mobility of both type of carriers decreases with increasing temperature. The ratio $n_{h} / n_{e}$ decrease above $50 \mathrm{~K}$. Above 

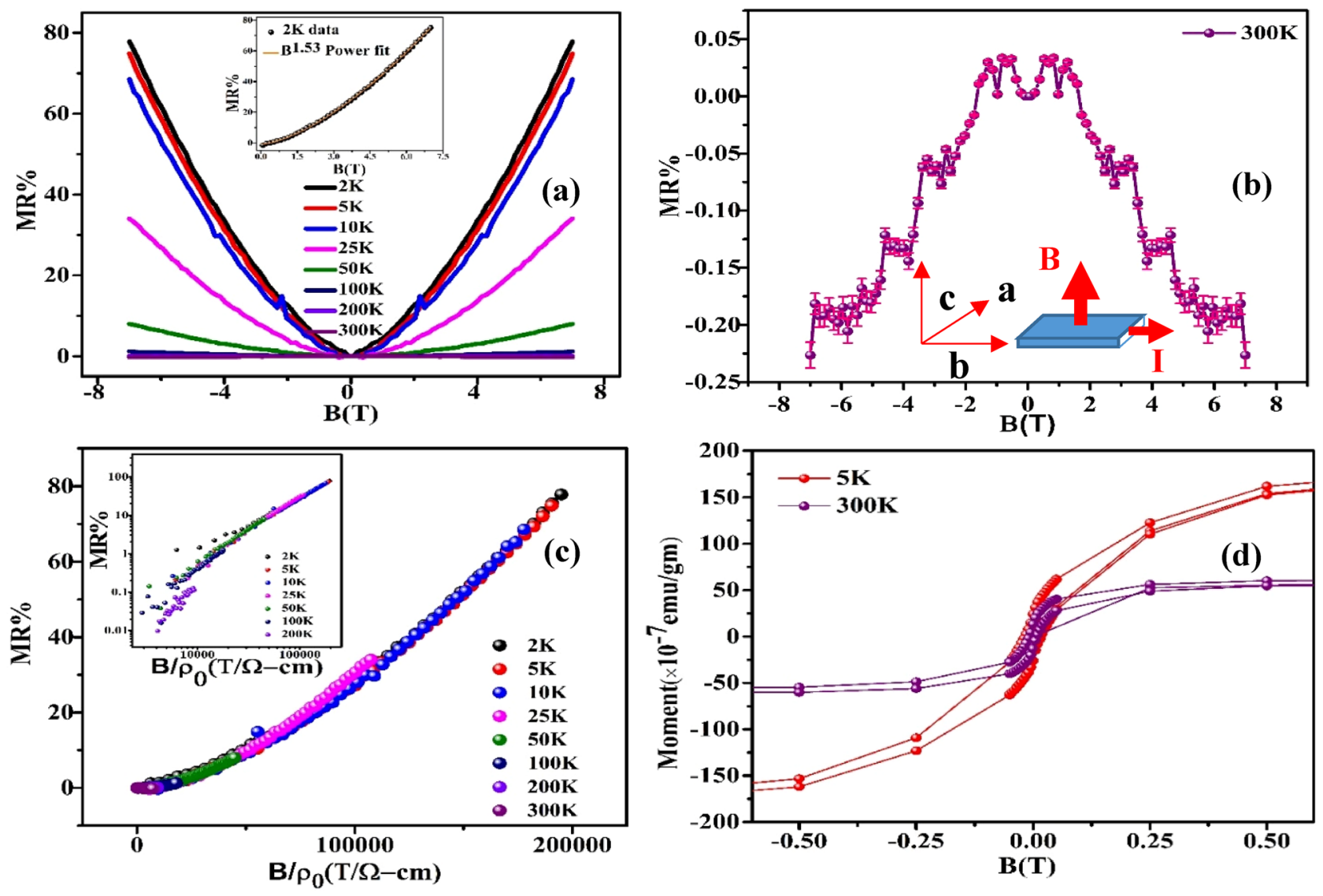

Figure 3. (a) Magnetic field dependent MR\% at various temperatures ranging from 2 to $300 \mathrm{~K}$, inset shows $2 \mathrm{~K}$ MR\% with a power law fit (orange solid line), (b) clear pictorial view of positive MR at low field and negative MR at high field for $300 \mathrm{~K}$ data, inset: measurement geometry, (c) Kohler's scaling of MR\% as a function of B/ $\rho_{0}$ up to $300 \mathrm{~K}$, inset: Kohler plot on logarithmic scale up to $200 \mathrm{~K}$, (d) M-B magnetic hysteresis curve taken at $5 \mathrm{~K}$ and $300 \mathrm{~K}$.

a certain temperature hole mobility becomes larger than electron mobility. Finally, at room temperature both the carriers are taking part in the transport. This might be the reason of S-shaped nature of Hall data at $300 \mathrm{~K}$.

It is worthwhile to mention that the large carrier mobility is decisive to the XMR effect in Weyl semimetal ${ }^{24}$. In the present case, in $\mathrm{T}_{\mathrm{d}}$-MoTeP we also observe (discussed above) the large carrier mobility. However, such colossal XMR effect is absent in the present system due to dominating ferromagnetic ordering effect (discussed below). The decreased mobility at high temperature can be expected as electron-phonon scattering is dramatically increased at high temperature.

Magnetization behavior. In order to find out the magnetic ordering we have also measured the magnetization behavior in MoTeP. Interestingly, we have found the presence of magnetic hysteresis in M(B) (Fig. 3d) indicating the ferromagnetic ordering in this compound. The magnitude of the loop decreases with increasing temperature. The estimated coercive fields are 278 and $195 \mathrm{Oe}$ at $5 \mathrm{~K}$ and $300 \mathrm{~K}$, respectively. This type of long range ordering is also reported in the pioneering work by Guguchia ${ }^{51}$ for the compound $2 \mathrm{H}-\mathrm{MoTe}_{2}$ and $\mathrm{MoSe}_{2}$. Such ferromagnetism was induced by defects like metal vacancies and chalcogen-metal antisites disorder. The Mo vacancy can also induce spin polarization with large magnetic moments ${ }^{22}$. Presence of vacancy $\left(V_{\text {Mo }}, V_{T e}\right.$, $\mathrm{V}_{\mathrm{P}}$ ) and antisite defects $\left(\mathrm{Te}_{\mathrm{p}}, \mathrm{Mo}_{\mathrm{Te}} \mathrm{Te}_{\mathrm{Mo}}\right.$ ) has been considered as most common point-defects in the 2D materials like $\mathrm{MoTe}_{2}{ }^{17}$.

Effect of magnetic field on longitudinal resistivity. The longitudinal resistivity $\rho_{\mathrm{xx}}$ measured under perpendicular current and magnetic field configuration. This $\rho_{\mathrm{xx}}$ follows a nearly quadratic dependence on magnetic field indicating no sign of saturation.

Magnetoresistance $\left[\mathrm{MR}=\left\{\rho_{\mathrm{xx}}(7 \mathrm{~T})-\rho_{\mathrm{xx}}(0 \mathrm{~T})\right\} / \rho_{\mathrm{xx}}(0 \mathrm{~T}), \rho_{\mathrm{xx}}\right.$ is the longitudinal resistivity] reaches $77.831 \%$ at $2 \mathrm{~K}$. This MR value is relatively small compared to the other reports on XMR family of compounds like $\mathrm{MoTe}_{2}$, $\mathrm{WTe}_{2}, \mathrm{MoP}_{2}$ and $\mathrm{WP}_{2}{ }^{34,43,44,52}$. Moreover, electron-hole compensation and electronic changes near $60 \mathrm{~K}(50 \mathrm{~K})$ was considered as the driving force behind the XMR in $\mathrm{MoTe}_{2}\left(\mathrm{WTe}_{2}\right)^{44,53}$. Particular orbital texture on the electron pocket was also proposed as a possible reason behind the XMR in $\mathrm{MoTe}_{2}{ }^{34}$. The $\mathrm{MR} \%$ value is gradually suppressed with increasing temperature. The MR\% decreases to $0.113 \%$ at about $200 \mathrm{~K}$ (Fig. 3b). A clear view of $300 \mathrm{~K} \mathrm{MR} \%$ data with error bar is represented in Fig. 3b. The positive MR weakened as the temperature is 


\begin{tabular}{|l|l|l|}
\hline Temperature & $\boldsymbol{\alpha}$ & Temperature (K) \\
\hline $\mathrm{MoTe}_{2}{ }^{65}$ & -0.41 to - 0.51 & $<2.5 \mathrm{~K}$ \\
\hline $\mathrm{MoTe}_{2}{ }^{66}$ & -0.8 & $1.5 \mathrm{~K}$ \\
\hline $\mathrm{WTe}_{2}{ }^{67}$ & $<<1$ & $2 \mathrm{~K}$ \\
\hline $\mathrm{MoSe}_{2}{ }^{68}$ & $0.56,0.49$ & - \\
\hline $\mathrm{MoTeP}$ (present work) & -0.449 & $2 \mathrm{~K}$ \\
\hline
\end{tabular}

Table 1. The values of $\alpha$ extracted from HLN fitting.

increased and finally the MR displayed mixed behavior from positive in low fields to negative in high fields when the temperature increased to $300 \mathrm{~K}$. However, all the data in Fig. $3 \mathrm{c}$ can be scaled onto a single line when MR plotted as a function of $\mathrm{B} / \rho_{0}$. However, deviation from the single line is observed above $50 \mathrm{~K}$ when plotted on $\log -\log$ scale (inset of 3c). Departure from the scaling signify the existence of both type of charge carrier in the system supporting the Hall data. Multiband effect with different scattering time was also considered as a reason behind the breakdown of Kohler's rule in $\mathrm{MoP}_{2}{ }^{54}$. The dominance of phonon scattering at high temperature might also be the reason of this deviation ${ }^{55}$. However, according to semi-classical two-band theory, the validity of Kohler's rule with $\mathrm{MR} \propto\left(\mathrm{B} / \rho_{0}\right)^{2}$ suggests an XMR or perfect electron-hole compensated system. Violation of Kohler's rule is common in XMR materials, such as $\mathrm{LaBi}$, TaAs, $\mathrm{TaAs}_{2}, \mathrm{NbAs}_{2}, \mathrm{NbSb}_{2}$, and $\mathrm{LaSbTe}^{49,56-59}$. Our trial to fit the Kohler's law $\left(M R=c\left(\frac{B}{\rho_{0}}\right)^{m}\right)$ yields $\mathrm{c}=25(\mu \Omega-\mathrm{cm} / \mathrm{T})^{1.65}$ and $\mathrm{m}=1.65$. The value of $\mathrm{m} \sim 1.65$ is away from a perfect electron-hole compensation $(\mathrm{m} \sim 2)$ situation. The deviation in Kohler's scaling is also in line with our nonlinear Hall data and multiband transport above $50 \mathrm{~K}$. We performed a power law (Fig. 3a inset) with $\mathrm{MR} \%$ data at $2 \mathrm{~K}$ and fitting yields $\mathrm{n}=1.53$ which shows a subquadratic field dependence ${ }^{60,61}$. MR $\sim \mathrm{B}^{\mathrm{n}}$ where $\mathrm{n}$ is predicted to be 2 for semimetals with perfect electron-hole compensation. Our fitted $\mathrm{n} \sim 1.53$ value also conveys the results of electron dominating transport at low temperature in accordance with the Hall data.

Nevertheless, the chiral anomaly induced negative LMR and positive transverse magnetoresistance (TMR) as a result of Lorentz force is fingerprint of the type-II Weyl semimetals ${ }^{37,42}$. In our case MoTeP also shows positive $\mathrm{MR}$ up to $200 \mathrm{~K}$ due to Lorentz force in perpendicular current and magnetic field configuration. The MR near $\mathrm{B}=0$ is also positive at $300 \mathrm{~K}$. On the other hand, under the application of external magnetic field the decrease in resistivity with increasing temperature is systematic in $\mathrm{T}_{\mathrm{d}}$-MoTeP up to $200 \mathrm{~K}$, as expected in semimetallic systems. The $2 \mathrm{~K}$ MR curve shows a small cusp-like feature at low field suggesting the presence of the weak antilocalization (WAL) effect. The conductivity change $\Delta \sigma=\Delta \sigma(\mathrm{B})-\Delta \sigma(0)$ arising from the quantum interference effects is explained by the Hikami-Larkin-Nagaoka (HLN) theory ${ }^{62}$ :

$$
\Delta \sigma=\sigma(B)-\sigma(0)=A\left[\psi\left(\frac{1}{2}+\frac{h}{8 \pi e B L_{\varphi}^{2}}\right)-\ln \left(\frac{h}{8 \pi e B L_{\varphi}^{2}}\right)\right],
$$

where $A=\frac{\alpha e^{2}}{\pi h}$ and $\alpha$ is a constant equal to 1 or $-1 / 2$ for weak localization or anti-localization respectively. $\psi$ is digamma function, $L_{\varphi}$ is the phase coherence length. We have calculated magnetoconductance per conduction channel $\Delta \sigma / Z^{\star}$ ( $Z^{\star}$ is no. of conduction layers). One $2 \mathrm{D}$ layer corresponds to $\mathrm{e}^{2} / \mathrm{h}$ conductance and that is equal to $2 \mathrm{QL}$ thickness i.e. $2 \mathrm{~nm}$. Therefore, $Z^{\star}$ would be equal to $t / 2 \mathrm{~nm}^{63}$ where $t$ is thickness of the sample. The $2 \mathrm{~K}$ data of $\Delta \sigma\left(\mathrm{e}^{2} / \mathrm{h}\right)$ shows a small phase coherence length, $\mathrm{L} \phi$, of about $29.98 \mathrm{~nm}$ indicating the weak WAL effect (Fig. 1d). The results are in accordance with previous WAL data in these family of compounds ${ }^{64}$. The calculated a value -0.449 confirms the WAL effect in the system. This quantum interference effect is significator of metallic state in the strong SOC system. This also indicates an enhanced spin scattering at this temperature. In addition to that, a negative a value confirms the WAL effect at low T and B range. At higher temperature WAL suppressed due to enhanced spin dependent scattering. The fitted values of $\mathrm{L}_{\Phi}$ are $29.98 \mathrm{~nm}\left(\mathrm{~A}=-0.14 \Omega^{-1}\right), 14.21 \mathrm{~nm}$ $\left(\mathrm{A}=-0.82 \Omega^{-1}\right), 10.28 \mathrm{~nm}\left(\mathrm{~A}=-1.96 \Omega^{-1}\right)$ and $5.38 \mathrm{~nm}\left(\mathrm{~A}=-6.73 \Omega^{-1}\right)$ at $2,5,10$ and $25 \mathrm{~K}$ respectively. The calculated value of $\alpha$ is compared with other reports on TMDs materials in Table 1.

Most interestingly, we have found negative magnetoresistance near room-temperature at high field. In a topological system few possibilities are there behind negative MR like-(a) chiral anomaly in Weyl semimetals, (b) current jetting effects, (c) weak localization effect, (d) ferromagnetism in the sample and (e) field induced magnetic impurity scattering. Observation of the chiral-anomaly induced negative MR requires the applied magnetic field to be parallel to the electric field ${ }^{47,69-71}$, which is not our case. An inhomogeneous distribution of the current flowing inside the sample can give rise to negative MR effect and current jetting ${ }^{72}$ shows strong dependence on sample geometry/size. However, in our case observed MR is systematically decreased to a negative value and is not observed in the whole range. Furthermore, this effect requires strong preference of the current to flow in the direction of the magnetic field ${ }^{73}$. This also rules out the possibility of current jetting effect in the present investigation. On the other hand, weak localization effects ${ }^{74,75}$ can cause negative magnetoresistance in impurity induced semimetals and semiconductors at low field. When two electron waves interfere constructively while travelling from opposite direction along a closed path, they scatter off by the impurity and leads to an increase in magneto conductivity. In our case, the magnetoconductivity decreases with increase in field, below $1 \mathrm{~T}$ indicating the effect of WAL. But at higher field at $300 \mathrm{~K}$ the observed negative MR cannot be due to the WAL.

It signifies that the magnetism plays an important role in the transition between positive and negative MR for $1 \mathrm{~T}^{\prime} \mathrm{MoTeP}$. The low-field positive MR becomes parabolic like at $300 \mathrm{~K}$. Under the application of external 

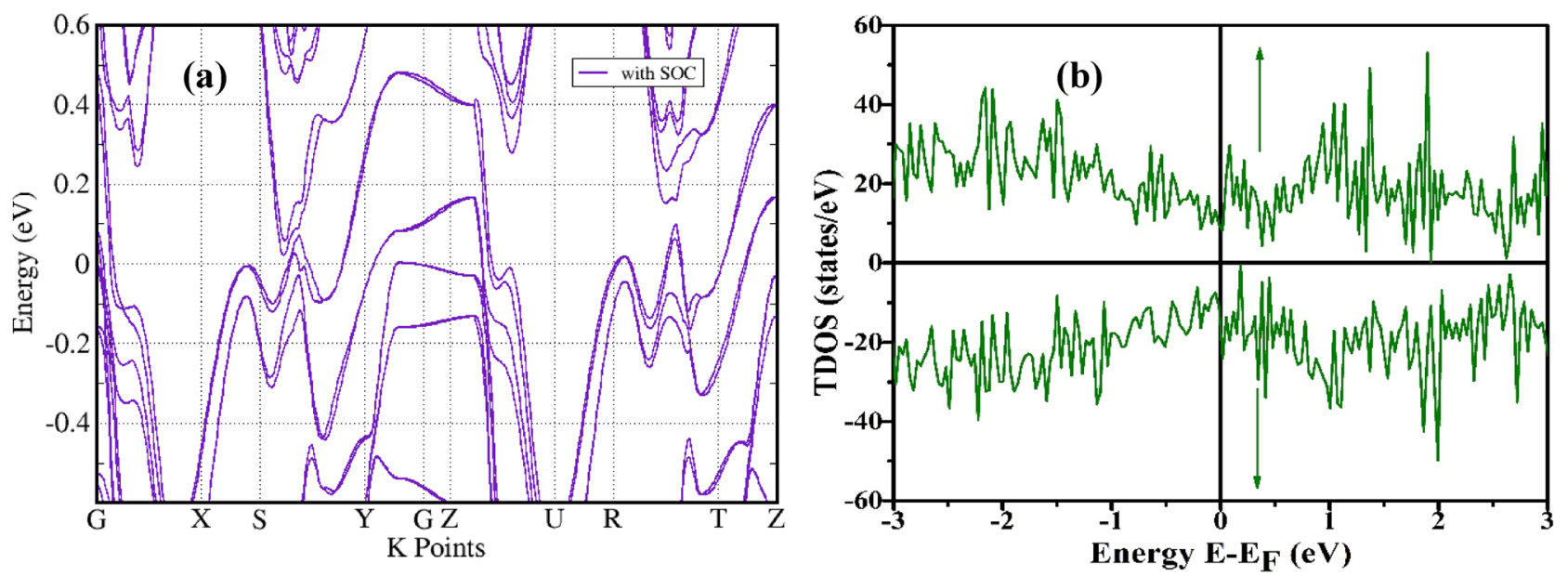

Figure 4. (a) Bulk band structure of $\mathrm{T}_{\mathrm{d}}-\mathrm{MoTeP}$ with inclusion of SOC, (b) Spin-resolved Total DOS for defect induced MoTeP.

magnetic field electronic scattering rate from local moments and impurities is suppressed resulting in increase in transport lifetime that results in a negative magnetoresistance. However, considering such effect for a particular field range is not appropriate rather we can expect the not so large overall MR effect is due to the ferromagnetism in the sample $\mathrm{e}^{73}$. Therefore, ferromagnetic ordering throughout the whole temperature range of measurement is the origin of low MR in the system. In fact, ferromagnetic ordering suppresses the scattering which in effect decreases the MR. Moreover, in Weyl semimetal the XMR decreases with increase of temperature and in the present case at $300 \mathrm{~K}$ the ferromagnetic ordering dominates over the scattering effect leading the negative MR.

In order to further determine the origin of FM ordering we have performed the spin polarized DOS calculation (Fig. 4b). It is observed that MoTeP exhibits semimetallic features in bandstructure (Fig. 4a) similar to parent $\mathrm{MoTe}_{2}{ }^{76}$. We speculate that the inclusion of SOC splits the hole and electron bands in two sets of hole and electron pockets with slightly different sizes. The hole bands are comparatively flatter than the electron bands in MoTeP indicating holes possess greater effective mass and smaller mobility than the electrons. This corroborates well with our experimental results. The TOTAL DOS and difference DOS between spin up and spin down contribution are illustrated in Fig. $4 \mathrm{~b}$ and S7 of supplementary. The asymmetric total DOS near the Fermi level associated with ferromagnetic behavior of the material. Furthermore, it is observed that Mo- $d$ states, Te- $p$ and P-p have the dominant contribution in their fat band calculations as shown in S5-S7 of supplementary. The total magnetic moment calculated is $2.414 \mu_{\mathrm{B}}$ with defect structure. Without producing defect, the moment is $0.0001 \mu_{\mathrm{B}}$ only. The defect produced moment is close to the magnetic moment calculated for other $\mathrm{MoX}_{2}$ compounds ${ }^{18}$. Interestingly, incorporation of defects in the crystal structure leads to ferromagnetic interaction from TDOS calculation which is consistent with those already reported ${ }^{17,18,21}$. The magnetism mainly promoted by Mo- $4 d$ orbital states.

\section{Conclusions}

We presented a systematic study of magnetotransport and magnetic properties of single-crystalline MoTeP. The semiclassical two-band fitting of the Hall and longitudinal conductivity explain near-perfect carrier compensation at low temperature with very high carrier mobilities. It is evident from the Hall resistivity data that the transport properties in $\mathrm{MoTeP}$ are dominated by electron-type charge carriers. The suppressed magnetoresistivity is the result of reduced scattering effects due to the defect induced ferromagnetism. Particularly, at room temperature, this scattering is again suppressed due to the applied high magnetic field. At room temperature $\rho_{\mathrm{yx}}$ becomes nonlinear at higher field, implying that both type of carriers is activated. Below $25 \mathrm{~K}$, the WAL-induced $\mathrm{MR}$ is extremely narrow within $1.5 \mathrm{~T}$. Kohler's scaling of $\mathrm{MR} \% \sim\left(\mathrm{B} / \rho_{0}\right)^{\mathrm{m}}$ with $\mathrm{m}=1.65$ together with a power law of $\mathrm{B}^{\mathrm{n}}$ where $\mathrm{n}=1.53$ supports the dominating electron charge carrier transport. Departure from linearity above $50 \mathrm{~K}$ interprets the temperature dependent variation of electron and hole charge carriers. Finally, at room temperature electron and hole joint transport is observed from nonlinear S-shaped Hall data. Importantly, ferromagnetic nature from the asymmetric spin polarized total DOS near Fermi level supports our experimental observation of defect induced ferromagnetic MoTeP. We found incorporation of dopants into the system explored many intriguing features and open up another avenue for future material science research.

Received: 27 October 2020; Accepted: 3 March 2021

Published online: 27 April 2021

\section{References}

1. Lv, B. Q. et al. Experimental discovery of Weyl semimetal TaAs. Phys. Rev. X 5, 031013 (2015).

2. Xu, S. Y. et al. Discovery of a Weyl fermion semimetal and topological Fermi arcs. Science 349, 613-617 (2015).

3. Deng, K. et al. Experimental observation of topological Fermi arcs in type-II Weyl semimetal MoTe 2. Nat. Phys. 12, 1105-1110 (2016). 
4. Huang, L. et al. Spectroscopic evidence for a type II Weyl semimetallic state in MoTe 2. Nat. Mater. 15, 1155-1160 (2016).

5. Jiang, J. et al. Signature of type-II Weyl semimetal phase in MoTe 2. Nat. Commun. 8, 1-6 (2017).

6. Wan, X., Turner, A. M., Vishwanath, A. \& Savrasov, S. Y. Topological semimetal and Fermi-arc surface states in the electronic structure of pyrochlore iridates. Phys. Rev. B 83, 205101 (2011).

7. Weng, H., Fang, C., Fang, Z., Bernevig, B. A. \& Dai, X. Weyl semimetal phase in noncentrosymmetric transition-metal monophosphides. Phys. Rev. X 5, 011029 (2015)

8. Sun, Y., Wu, S.-C., Ali, M. N., Felser, C. \& Yan, B. Prediction of Weyl semimetal in orthorhombic MoTe 2. Phys. Rev. B 92, 161107 (2015).

9. Wu, Y. et al. Observation of Fermi arcs in the type-II Weyl semimetal candidate WTe 2. Phys. Rev. B 94, 121113 (2016).

10. Chang, T. R. et al. Prediction of an arc-tunable Weyl Fermion metallic state in MoxW1-xTe2. Nat. Commun. 7, 1-9 (2016).

11. Autès, G., Gresch, D., Troyer, M., Soluyanov, A. A. \& Yazyev, O. V. Robust type-II Weyl semimetal phase in transition metal diphosphides X P 2 ( X = Mo, W). Phys. Rev. Lett. 117, 066402 (2016).

12. Nielsen, H. B. \& Ninomiya, M. The Adler-Bell-Jackiw anomaly and Weyl fermions in a crystal. Phys. Lett. B 130, 389-396 (1983).

13. Son, D. T. \& Spivak, B. Z. Chiral anomaly and classical negative magnetoresistance of Weyl metals. Phys. Rev. B 88, 104412 (2013).

14. Wang, Y. et al. Gate-tunable negative longitudinal magnetoresistance in the predicted type-II Weyl semimetal WTe 2. Nat. Commun. 7, 1-6 (2016).

15. Lv, Y.-Y. et al. Experimental observation of anisotropic adler-bell-jackiw anomaly in type-II Weyl semimetal WTe 1.98 crystals at the quasiclassical regime. Phys. Rev. Lett. 118, 096603 (2017).

16. Liang, D. D. et al. Origin of planar Hall effect in type-II Weyl semimetal MoTe2. AIP Adv. 9, 55015 (2019).

17. Li, Y. et al. The magnetism of intrinsic structural defects in monolayer MoTe2. J. Alloys Compd. 735, 2363-2372 (2018).

18. Ma, Y. et al. Electronic and magnetic properties of perfect, vacancy-doped, and nonmetal adsorbed MoSe2, MoTe2 and WS2 monolayers. Phys. Chem. Chem. Phys. 13, 15546-15553 (2011).

19. Huang, H. H. et al. Controlling phase transition for single-layer MTe2 ( $\mathrm{M}=\mathrm{Mo}$ and W): Modulation of the potential barrier under strain. Phys. Chem. Chem. Phys. 18, 4086-4094 (2016).

20. Lin, X. \& Ni, J. Magnetism and electronic phase transitions in monoclinic transition metal dichalcogenides with transition metal atoms embedded. J. Appl. Phys. 120, 064305 (2016).

21. Han, S. W. et al. Electron beam-formed ferromagnetic defects on MoS2 surface along 1 T phase transition. Sci. Rep. 6, 38730 (2016).

22. Kanoun, M. B. Tuning magnetic properties of two-dimensional MoTe2 monolayer by doping $3 \mathrm{~d}$ transition metals: Insights from first principles calculations. J. Alloys Compd. 748, 938-942 (2018).

23. Hu, A.-M., Wang, L., Xiao, W.-Z. \& Meng, B. Electronic structures and magnetic properties in Cu-doped two-dimensional dichalcogenides. Phys. E Low-dimensional Syst. Nanostruct. 73, 69-75 (2015).

24. Zhou, Q. et al. Hall effect within the colossal magnetoresistive semimetallic state of MoTe2. Phys. Rev. B 94, 1-5 (2016).

25. Paul, S. et al. Tailoring the phase transition and electron-phonon coupling in $1 \mathrm{~T}^{\prime}-\mathrm{MoTe} 2$ by charge doping: A Raman study. Phys. Rev. B 102, 054103 (2020).

26. Qi, Y. et al. Superconductivity in Weyl semimetal candidate MoTe2. Nat. Commun. 7, 11038 (2016).

27. Espejo, C., Rangel, T., Pouillon, Y., Romero, A. H. \& Gonze, X. Wannier functions approach to van der Waals interactions in ABINIT. Comput. Phys. Commun. 183, 480-485 (2012).

28. Turner, P. J. XMGRACE, Version 5.1. 19. Cent. Coast. Land-Margin Res. Oregon Grad. Inst. Sci. Technol. Beaverton, OR (2005).

29. Shrestha, K. et al. Extremely large nonsaturating magnetoresistance and ultrahigh mobility due to topological surface states in the metallic Bi2Te3 topological insulator. Phys. Rev. B 95, 195113 (2017).

30. Hu, J. et al. $\pi$ Berry phase and Zeeman splitting of Weyl semimetal TaP. Sci. Rep. 6, 18674 (2016).

31. Xiang, Z. J. et al. Angular-Dependent Phase Factor of Shubnikov-de Haas Oscillations in the Dirac Semimetal Cd3As2. Phys. Rev. Lett. 115, 226401 (2015).

32. Berger, A. N. et al. Temperature-driven topological transition in $1 \mathrm{~T}^{\prime}$-MoTe2. npj Quantum Mater. 3, 2 (2018).

33. Yan, X. J. et al. Investigation on the phase-transition-induced hysteresis in the thermal transport along the c-axis of MoTe2. $n p j$ Quantum Mater. 2, 1-7 (2017).

34. Chen, F. C. et al. Extremely large magnetoresistance in the type-II Weyl semimetal Mo Te 2. Phys. Rev. B 94, 235154 (2016).

35. Wu, Y. et al. Temperature-Induced Lifshitz Transition in WTe 2. Phys. Rev. Lett. 115, 166602 (2015).

36. Lim, S., Rajamathi, C. R., Süß, V., Felser, C. \& Kapitulnik, A. Temperature-induced inversion of the spin-photogalvanic effect in WTe 2 and MoTe 2. Phys. Rev. B 98, 121301 (2018).

37. Pei, Q. L. et al. Origin of the turn-on phenomenon in T d - MoT e 2. Phys. Rev. B 96, 075132 (2017).

38. Soluyanov, A. A. et al. Type-II Weyl semimetals. Nature 527, 495-498 (2015).

39. Chang, T.-R. et al. Prediction of an arc-tunable Weyl Fermion metallic state in MoxW1-xTe2. Nat. Commun. 7, 10639 (2016).

40. Wang, Y. L. et al. Origin of the turn-on temperature behavior in WTe 2. Phys. Rev. B 92, 180402 (2015).

41. Lv, Y.-Y. et al. Mobility-controlled extremely large magnetoresistance in perfect electron-hole compensated a - W P 2 crystals. Phys. Rev. B 97, 245151 (2018).

42. Kumar, N. et al. Extremely high magnetoresistance and conductivity in the type-II Weyl semimetals WP2 and MoP2. Nat. Commun. 8, 1642 (2017).

43. Luo, Y. et al. Hall effect in the extremely large magnetoresistance semimetal WTe 2 . Appl. Phys. Lett. 107, 182411 (2015).

44. Jha, R., Higashinaka, R., Matsuda, T. D., Ribeiro, R. A. \& Aoki, Y. Anomalous magnetotransport properties of high-quality single crystals of Weyl semimetal WTe2: Sign change of Hall resistivity. Phys. B Condens. Matter 536, 68-71 (2018).

45. Vu, D. M. et al. Weak antilocalization and two-carrier electrical transport in B i $1-\mathrm{x} \mathrm{S} \mathrm{b} \mathrm{x} \mathrm{single} \mathrm{crystals}(0 \% \leq \mathrm{x} \leq 17.0 \%)$. Phys. Rev. B 100, 125162 (2019).

46. Narayanan, A. et al. Linear magnetoresistance caused by mobility fluctuations in n-doped Cd 3 As 2. Phys. Rev. Lett. 114, 117201 (2015).

47. Zheng, G. et al. Transport evidence for the three-dimensional Dirac semimetal phase in ZrT e 5. Phys. Rev. B 93, 115414 (2016).

48. Jo, N. H., Wang, L. L., Orth, P. P., Bud'ko, S. L. \& Canfield, P. C. Magnetoelastoresistance in WTe2: Exploring electronic structure and extremely large magnetoresistance under strain. Proc. Natl. Acad. Sci. USA 116, 25524-25529 (2019).

49. Singha, R., Pariari, A., Satpati, B. \& Mandal, P. Magnetotransport properties and evidence of a topological insulating state in LaSbTe. Phys. Rev. B 96, 245138 (2017).

50. Singha, R., Roy, S., Pariari, A., Satpati, B. \& Mandal, P. Planar Hall effect in the type-II Dirac semimetal VAl 3. Phys. Rev. B 98, 081103 (2018).

51. Guguchia, Z. et al. Magnetism in semiconducting molybdenum dichalcogenides. Sci. Adv. 4, eaat3672 (2018).

52. Ali, M. N. et al. Large, non-saturating magnetoresistance in WTe2. Nature 514, 205-208 (2014).

53. Lee, S. et al. Origin of extremely large magnetoresistance in the candidate type-II Weyl semimetal MoTe2-x. Sci. Rep. 8, 1-8 (2018).

54. Wang, A. et al. Magnetotransport properties of MoP 2. Phys. Rev. B 96, 195107 (2017).

55. Huang, S.-M., Yu, S.-H. \& Chou, M. The temperature dependence of the crossover magnetic field of linear magnetoresistance in the $\mathrm{Cu}_{0.1} \mathrm{Bi}_{2} \mathrm{Se}_{3}$. Mater. Res. Express 3, 086103 (2016).

56. Wang, K., Graf, D., Li, L., Wang, L. \& Petrovic, C. Anisotropic giant magnetoresistance in NbSb2. Sci. Rep. 4, 7328 (2014).

57. Yuan, Z., Lu, H., Liu, Y., Wang, J. \& Jia, S. Large magnetoresistance in compensated semimetals TaAs 2 and NbAs 2. Phys. Rev. B 93, 184405 (2016). 
58. Sun, S., Wang, Q., Guo, P.-J., Liu, K. \& Lei, H. Large magnetoresistance in LaBi: Origin of field-induced resistivity upturn and plateau in compensated semimetals. New J. Phys. 18, 082002 (2016).

59. Zhang, C.-L. et al. Electron scattering in tantalum monoarsenide. Phys. Rev. B 95, 085202 (2017).

60. Fatemi, V. et al. Magnetoresistance and quantum oscillations of an electrostatically tuned semimetal-to-metal transition in ultrathin WTe 2. Phys. Rev. B 95, 041410 (2017).

61. Li, P. et al. Anisotropic planar Hall effect in the type-II topological Weyl semimetal WTe 2. Phys. Rev. B 100, 205128 (2019).

62. Hikami, S., Larkin, A. I. \& Nagaoka, Y. Spin-orbit interaction and magnetoresistance in the two dimensional random system. Prog. Theor. Phys. 63, 707-710 (1980).

63. Shrestha, K., Graf, D., Marinova, V., Lorenz, B. \& Chu, C. W. Weak antilocalization effect due to topological surface states in $\mathrm{Bi}_{2}$ $\mathrm{Se}_{2,1} \mathrm{Te}_{0.9}$. J. Appl. Phys. 122, 145901 (2017).

64. Mathew, R. J. et al. High unsaturated room-temperature magnetoresistance in phase-engineered MoxW1-xTe2+ $\delta$ ultrathin films. J. Mater. Chem. C 7, 10996-11004 (2019).

65. Wang, Q. et al. Observation of weak anti-localization and electron-electron interaction on few-layer $1 \mathrm{~T}^{\prime}$-MoTe2 thin films. Chin. Phys. Lett. 35, 1-4 (2018).

66. Gan, Y. et al. Bandgap opening in MoTe2 thin flakes induced by surface oxidation. Front. Phys. 15, 1-7 (2020).

67. Naylor, C. H. et al. HHS Public Access. Vol. 4, (2018).

68. Papadopoulos, N., Watanabe, K., Taniguchi, T., Van Der Zant, H. S. J. \& Steele, G. A. Weak localization in boron nitride encapsulated bilayer MoS2. Phys. Rev. B 99, 1-5 (2019).

69. Wang, Z. et al. Helicity-protected ultrahigh mobility Weyl fermions in NbP. Phys. Rev. B 93, 121112 (2016).

70. Xiong, J. et al. Evidence for the chiral anomaly in the Dirac semimetal $\mathrm{Na}_{3} \mathrm{Bi}$. Science 350, 413-416 (2015).

71. Li, H. et al. Negative magnetoresistance in Dirac semimetal Cd3As2. Nat. Commun. 7, 10301 (2016).

72. Pippard, A. B. Magnetoresistance in Metals. (1989).

73. Coleman, R. V. \& Isin, A. Magnetoresistance in iron single crystals. J. Appl. Phys. 37, 1028-1029 (1966).

74. Baxter, D. V., Richter, R., Trudeau, M. L., Cochrane, R. W. \& Strom-Olsen, J. O. Fitting to magnetoresistance under weak localization in three dimensions. J. Phys. 50, 1673-1688 (1989).

75. Kawabata, A. Theory of negative magnetoresistance I. Application to heavily doped semiconductors. J. Phys. Soc. Jpn. 49, 628-637 (1980).

76. Guguchia, Z. et al. Signatures of the topological s+- superconducting order parameter in the type-II Weyl semimetal Td-MoTe2. Nat. Commun. 8, 1082 (2017).

\section{Acknowledgements}

DP is grateful to MHRD for providing fellowship. DP is also grateful to CIFC, IIT(BHU) for providing MFM facility. The support and the resources provided by 'PARAM Shivay Facility' under the National Supercomputing Mission, Government of India at the Indian Institute of Technology, Varanasi are gratefully acknowledged. SP acknowledges the financial support from DST-SERB, India under the project no. ECR/2017/001243. PS is also gratetful to DST India.

\section{Author contributions}

D.P. wrote the main manuscript, S.K., P.S. and A.V. conducted the experiments, S.D., V. K.G. and M.S. reviewed the manuscript. S.C., Y.U. and S.S. provided the magnetic, transport and Raman facilities; S.P. and S. C. supervised and revised the manuscript. All authors reviewed the manuscript.

\section{Competing interests}

The authors declare no competing interests.

\section{Additional information}

Supplementary Information The online version contains supplementary material available at https://doi.org/ 10.1038/s41598-021-88669-8.

Correspondence and requests for materials should be addressed to S.P. or S.C.

Reprints and permissions information is available at www.nature.com/reprints.

Publisher's note Springer Nature remains neutral with regard to jurisdictional claims in published maps and institutional affiliations.

Open Access This article is licensed under a Creative Commons Attribution 4.0 International format, as long as you give appropriate credit to the original author(s) and the source, provide a link to the Creative Commons licence, and indicate if changes were made. The images or other third party material in this article are included in the article's Creative Commons licence, unless indicated otherwise in a credit line to the material. If material is not included in the article's Creative Commons licence and your intended use is not permitted by statutory regulation or exceeds the permitted use, you will need to obtain permission directly from the copyright holder. To view a copy of this licence, visit http://creativecommons.org/licenses/by/4.0/.

(C) The Author(s) 2021 